\title{
Uma abordagem longitudinal da contribuição do trauma e da vergonha nos sintomas depressivos em adolescentes*
}

A longitudinal approach to the contribution of trauma and external shame on depressive symptoms in adolescence

Artigo Original | Original Article

\author{
Marina Cunha PhD (1,2a), Rute Almeida PsyM (1b), Sónia Cherpe PsyM (2c), Sónia Simões PhD (1d), Mariana Marques PhD (1d) \\ (1) Instituto Superior Miguel Torga (ISMT), Coimbra, Portugal \\ (2) Centro de Investigação em Neuropsicologia e Intervenção Cognitivo-comportamental (CINEICC), FPCE da Universidade de Coimbra; Portugal. \\ (a) Elaboração do desenho de investigação; Tratamento estatístico dos dados; análise dos resultados; Revisão do trabalho. \\ (b) Execução do trabalho; Recolha e inserção dos dados. \\ (c) Elaboração do desenho de investigação; Definição de procedimentos; Acesso à amostra. \\ (d) Revisão do trabalho.
}

Autor para correspondência | Corresponding author: Marina Isabel Vieira Antunes da Cunha; Rua Augusta, 46, 3000-061, Coimbra; marina_cunha@ismt.pt

* Este artigo resulta de uma dissertação de mestrado em Psicologia Clínica e uma versão breve desta investigação foi alvo de um póster apresentado nas $2^{\mathrm{a}} \mathrm{s}$ Jornadas de Investigação em Psicologia Clínica do Instituto Superior Miguel Torga, tendo ganho o $1^{\circ}$ prémio atribuído por um júri qualificado para o efeito.

\section{Palavras-Chave}

Depressão

Adolescentes

Acontecimentos traumáticos

Sentimentos de vergonha

Estudo longitudinal

\section{RESUMO}

Contexto: A revisão da literatura sobre potenciais fatores preditores dos sintomas depressivos em adolescentes tem mostrado que as experiências traumáticas durante a infância, as experiências de vergonha e o género têm um contributo relevante.

Objetivo: Pretende-se com o presente estudo observar a variabilidade intraindividual da vergonha, acontecimentos traumáticos e género e testar o poder preditivo destas variáveis a 6 meses na evolução de sintomas depressivos (variável dependente) em adolescentes.

Método: A amostra foi constituída por 325 adolescentes, com idades compreendidas entre os 12 e os 18 anos, distribuídos pela zona centro de Portugal e a frequentar o $3 .^{\circ}$ ciclo do ensino básico e ensino secundário. Foram utilizados o Inventário de Depressão para Crianças, a Escala Breve de vergonha e o Questionário de Trauma na Infância para a avaliação das variáveis referidas. Os resultados longitudinais foram analisados através de uma análise de regressão linear múltipla.

Resultados: Verificou-se uma associação positiva entre experiências relatadas como traumáticas e as perceções de vergonha (T1) e os sintomas depressivos (T2, após 6 meses). O modelo de regressão linear múltipla explicou $63 \%$ da variância dos sintomas depressivos no T2, podendo contemplar-se que a pertença ao género feminino, a experiência de sentimentos de vergonha e de acontecimentos percebidos como abuso afetivo, abuso sexual e de negligência emocional (variáveis do trauma) permitiram predizer sintomas depressivos na adolescência.

Conclusão: Dado que existe alguma evidência do impacto de acontecimentos traumáticos do tipo abuso/negligência durante a infância e de perceções de vergonha, durante a adolescência no desenvolvimento de sintomas depressivos, será pertinente que estas variáveis sejam tidas em conta, quer na avaliação, quer nas intervenções psicoterapêuticas nesta etapa do desenvolvimento humano. Este estudo contribui para salientar o papel de fatores de vulnerabilidade para os sintomas depressivos na adolescência.

Keywords

\section{Depression}

Adolescents

Traumatic events

Shame feelings

Longitudinal study

\section{ABSTRACT}

Background: The review of the literature on potential predictors of depressive symptoms has shown that traumatic experiences during childhood, experiences of shame, and gender have a relevant contribution.

Aim: This study aimed to observe the intra-individual variability of shame, traumatic events, and gender and to test the predictive power of these variables in the evolution of depressive symptoms (dependent variable) at six months, in adolescents.

Method: The sample consisted of 325 adolescents, aged between 12 and 18 years old, distributed in the center of Portugal and attending the secondary/high school. The Children's Depression Inventory, the Brief Scale of Shame, and the Childhood Trauma Questionnaire were used to assess the listed variables. Longitudinal results were analyzed by multiple linear regression analysis.

Results: There was a positive association between experiences reported as traumatic and perceptions of shame (T1) and depressive symptoms (T2, after six months). The multiple linear regression model explained $63 \%$ of the depressive symptoms' variance at T2, and belonging to the female gender, the experience of shame and perceived events of emotional and sexual abuse, as emotional neglect (variables of the trauma) seems to predict depressive symptoms in adolescence.

Conclusions: Given that there is some evidence of the impact of traumatic events of childhood abuse/neglect, and perceptions of shame during adolescence on the evolution of depressive symptoms, it is relevant that these variables are considered in the assessment and in the psychotherapeutic interventions at this stage of human development. This study contributes to highlight the role of vulnerability factors for the depressive symptoms in adolescence. 


\section{INTRODUÇÃO}

As experiências adversas na infância, tal como exposição a acontecimentos traumáticos e vivências de vergonha podem moldar a forma como os adolescentes se percecionam a si próprios e aos outros, bem como lidam com as adversidades, podendo aumentar a sua vulnerabilidade para a perturbação depressiva. $\mathrm{Na}$ verdade, alguns estudos mostraram que as experiências traumáticas na infância e na adolescência, sentimentos de vergonha e o género têm sido consideradas variáveis preditoras de sintomatologia depressiva (Cunha, Matos, Faria, \& Zagalo, 2012; Matos, Pinto-Gouveia, \& Duarte, 2013; Monteiro, Matos, \& Oliveira, 2015). Este tipo de vivências de caráter traumático encontra-se associado a um maior risco de episódios de vergonha, que por sua vez aumentam o risco de sintomatologia depressiva (Bennett, Sullivan, \& Lewis, 2010; Muris et al., 2016).

O período de transição da adolescência, consensualmente considerado como um período de mudanças acentuadas a nível físico e psicológico, é caraterizado pela emergência de competências cognitivas e socio-emocionais que vão refletir-se na aquisição de novos papéis e responsabilidades pessoais e sociais por parte do jovem, bem como propiciar uma maior exposição a diversos fatores indutores de stresse. Estas características tornam este período especialmente desafiante, o qual inclui potencialidades, mas também vulnerabilidades próprias (Dahl, 2004; Steinberg, 2010). Enquanto a maioria dos jovens pode reagir positivamente a estas mudanças típicas da adolescência, existe igualmente um número considerável de indivíduos que desenvolve dificuldades ou problemas de adaptação que interferem negativamente nas várias áreas da vida (social, escolar, familiar, individual) (Barry, Murphy, \& O’Donovan, 2017). Ao nível da saúde mental, alguns estudos têm revelado um aumento da prevalência das dificuldades emocionais (de carácter exteriorizado e internalizado) na adolescência (e.g., Costello, Copeland, \& Angold, 2011; Thapar, Collishaw, Pine, \& Thapar, 2012; World Health Organization, 2018). Especificamente a sintomatologia depressiva e/ou depressão é uma das condições mais frequentes na adolescência (Gore et al., 2011), sabendo-se, por exemplo, que os principais sintomas depressivos aumentam cerca de $2 \%$ no início da adolescência (1315anos) e 15\% na adolescência média (15-18anos) (Ahmed, Bittencourt-Hewitt, \& Sebastian, 2015). Neste sentido, estudos que procurem clarificar os fatores de desenvolvimento e ou de manutenção dos sintomas depressivos, bem como a relação entre estes fatores, enquanto moderadores ou mediadores, podem desempenhar um papel fundamental na identificação precoce dos jovens em risco, contribuindo, assim, para a prevenção do desenvolvimento ou agravamento desta perturbação.

O estudo de Shore, Toumbourou, Lewis e Kremer (2018) sobre trajetórias longitudinais de adolescentes com sintomas depressivos mostrou que sintomas fracos a moderados estavam associados a um ajustamento psicossocial pobre dos jovens (traduzido por diversos indicadores). Este estudo identificou o género, o estatuto socioeconómico, acontecimentos de vida indutores de stresse, problemas de comportamento, uso de substâncias e problemas nas relações com os pais e pares como fatores preditores das trajetórias de maior sintomatologia depressiva (Shore et al, 2018). Na revisão da literatura, os acontecimentos traumáticos na infância têm sido consensualmente considerados um fator de risco para o desenvolvimento de psicopatologia depressiva em idade adulta. Os indivíduos com história de abuso sexual na infância relatam uma história de vida de depressão em cerca de 30-40\% (Mandelli, Petrelli, \& Serretti, 2015; Musliner \& Singer, 2014). Outros tipos de traumas ocorridos ao longo da infância e adolescência (e.g., abuso emocional, negligência física, emocional) têm mostrado poder operar como fatores de risco para uma variedade de perturbações mentais que persistem na idade adulta (Firth, 2014; Liu, Alloy, Abramson, lacoviello, \& Whitehouse, 2009; Mandelli et al., 2015). Crianças com história de abandono/negligência estão em risco de aumento de sintomas depressivos na idade adulta (Hopwood, Ansell, Fehon, \& Grilo, 2011; Kim \& Cicchetti, 2006; Pinquart \& Shen, 2011).

Tolchinsky (2014) define que o trauma é um fenómeno biopsicossocial complexo, que envolve a exposição a múltiplos eventos traumáticos, de natureza interpessoal, durante a infância que resulta em consequências adversas. Estas experiências emocionais negativas estão muitas vezes associadas a vivências acentuadas de vergonha e raiva, podendo a vergonha tornar-se parte da identidade da vítima, moldando a forma como se perceciona a si mesmo e aos outros, com efeitos nocivos na saúde mental ao longo da vida (Negrao, Bonanno, Noll, Putnam, \& Trickett, 2005; Stuewing \& McCloskey, 2005).

Por sua vez, a vergonha é conceptualizada por diversos autores como uma emoção multifacetada, autoconsciente, relacionada com uma avaliação global negativa do indivíduo acerca de si mesmo que influencia 
de forma poderosa o funcionamento psicológico ao longo da vida, a nível individual, interpessoal e cultural (Gilbert, 1998; Matos \& Pinto-Gouveia, 2010; Tangney \& Dearing, 2002).

Segundo o modelo biopsicossocial defendido por Gilbert (2002, 2006), a emoção de vergonha tem um papel fundamental na vida do individuo já que vai moldar o seu comportamento em contextos sociais, influenciar os sentimentos envolventes na relação consigo próprio e contribuir na construção da sua identidade, aceitabilidade e desejabilidade social (Matos \& Pinto-Gouveia, 2010; Tangney \& Dearing, 2002). Com efeito, Gilbert defende que na base da vergonha estão avaliações e sentimentos focados na forma como o eu é visto e julgado pelos outros, no eu como objeto, e no eu julgado pelo eu. Assim sendo, apesar de relacionadas, podem ser distinguidas duas dimensões da vergonha: externa e interna. A vergonha externa diz respeito à perceção que o individuo tem sobre a forma como os outros o vêm. Concretamente, o indivíduo receia ser visto pelos outros como inferior, defeituoso ou incompetente e, consequentemente, que esta visão negativa possa levar à rejeição, ataque ou perda da atratividade aos olhos dos outros (Gilbert, 2006). Por sua vez, a vergonha interna refere-se a uma visão negativa por parte do sujeito em relação a si mesmo, que o leva sentir-se inferior e inadequado, na comparação com os outros, mesmo quando estes manifestam uma avaliação e afeto positivo pelo indivíduo. Neste caso, o foco de atenção está no eu e não no mundo social externo e dirigido para a mente do outro. Contudo, tal como afirmámos anteriormente, estas duas dimensões estão amplamente associadas, já que ambas se reportam a atributos negativos do eu e se potenciam mutuamente (Goss, Gilbert, \& Allan, 1994). A emoção de vergonha (quer interna, quer externa) tem sido descrita como associada à psicopatologia (Feiring, 2005; SzentágotaiTătar et al., 2015), nomeadamente ao desenvolvimento e manutenção de sintomas depressivos, quer em adultos (e.g., Matos \& Pinto-Gouveia, 2010; Matos et al., 2013), quer em adolescentes (Åslund, Nilsson, Starrin, \& Sjöberg, 2007; Cunha et al., 2012; Irons \& Gilbert, 2005; Kim, Talbot, \& Cicchetti, 2009). É possível compreender que os aspetos da vergonha parecem especialmente relevantes na infância e adolescência, tendo em conta que neste período as competições sociais se podem tornar mais intensas e relevantes, orientadas para a construção de autoidentidade (Gilbert \& Irons, 2009; McLean, Breen, \& Fournier, 2010; Steinberg, 2005).

Tendo em conta o conhecimento atual sobre a relevância dos fatores de risco na sintomatologia depressiva, o presente estudo procura compreender o poder preditivo dos acontecimentos de vida traumáticos e experiências de vergonha na evolução ao longo de seis meses da sintomatologia depressiva em adolescentes, controlando o efeito do género.

\section{MÉTODO}

\section{Participantes}

Participaram inicialmente neste estudo (T1) 401 adolescentes, 239 do sexo feminino e 162 do sexo masculino, a frequentar o $3 .^{\circ}$ ciclo do ensino básico e ensino secundário, em escolas da zona centro de Portugal, com idades compreendidas entre os 13 e os 17 anos.

No segundo momento de avaliação (T2), seis meses depois, verificou-se uma perda de 76 indivíduos (19\%), sendo esta perda explicada pela mudança da escola por parte dos jovens, pela dificuldade de os contactar ou pela desistência de participação no estudo. A amostra final, relativa ao estudo longitudinal, ficou, assim, constituída por 325 indivíduos (199 do sexo feminino e 126 do sexo masculino). As idades variaram entre os 13 e os 18 anos ( $M=14,50 ; D P=1,28)$, não se verificando diferenças significativas em função do $\operatorname{sexo}\left(t_{(269,93)}=\right.$ $0,27 ; p=0,789)$.

\section{Instrumentos}

Para além da recolha de informação relativa às características sociodemográficas, nomeadamente sexo, idade e ano de escolaridade a frequentar, foi utilizado um conjunto de questionários de autorresposta para avaliar as variáveis de interesse.

Inventário de Sintomas Depressivos para crianças (Children's Depression Inventory - CDII; Kovacs, 1983; tradução e adaptação portuguesa: Marujo, 1994). Este instrumento é constituído por 27 itens distribuídos por vários grupos de sentimentos e ideias, cada um com três alternativas de resposta, com o intuito de avaliar como a criança/adolescente descreve os seus sentimentos nas duas últimas duas semanas. As afirmações são classificadas em ordem crescente de gravidade e a pontuação varia entre 0 - "ausência de sintomas" e 2 "sintoma definitivo". A pontuação total pode variar entre 0 e 54 pontos e os resultados permitem estabelecer uma definição empírica de sintomatologia depressiva (Cardoso, Rodrigues, \& Vilar, 2004). Este inventário engloba cinco dimensões, sendo estas o humor negativo, problemas interpessoais, ineficácia, anedonia e autoestima negativa. 
A versão portuguesa deste inventário, à semelhança da versão original americana, revelou igualmente uma boa consistência interna (apresentando coeficientes de alfa de Cronbach que variaram entre 0,80 e 0,84 ) para o total da escala (Pereira, Matos, \& Azevedo, 2014). No presente estudo, o total do questionário revelou uma excelente consistência interna, quer no T1 $(\alpha$ de Cronbach $=0,90)$, como no T2 $(\alpha$ de Cronbach $=0,89$ ).

Questionário de Trauma para a Infância (Childhood Trauma Questionnaire - CTQ; Bernstein \& Fink, 2004; tradução e adaptação: Cherpe, Matos, \& Pereira, 2010). É um instrumento de autorresposta que avalia as experiências de abuso e negligência da criança ao longo do seu desenvolvimento. Inclui 28 itens, 25 dos quais procuram avaliar, em termos retrospetivos, as cinco formas de abuso sugeridas por Bernstein e colaboradores (2003), o abuso afetivo, abuso físico, abuso sexual, a negligência emocional e a negligência física. Os restantes três itens avaliam a tendência de negação ou de minimização do abuso (Bernstein et al., 2003). Para cada item é apresentada uma escala de resposta de 5 pontos, as pontuações variam de 1 "nunca verdadeiro" a 5 "muito frequente". O estudo original demonstrou uma boa consistência interna nos cinco fatores com valores de alfa de Cronbach entre 0,61 e 0,95 (Bernstein et al., 2003; Spinhoven et al., 2014), assim como a versão portuguesa (Grassi-Oliveira, Stein, \& Pezzi, 2006). No presente estudo demonstrou igualmente uma consistência interna adequada no $\mathrm{T} 1$ e T2, nos seguintes fatores: abuso afetivo ( $\alpha$ de Cronbach $=0,70$ e $\alpha$ de Cronbach $=0,77$, respetivamente); abuso físico ( $\alpha$ de Cronbach $=0,70$ e $\alpha$ de Cronbach $=0,71$; abuso sexual ( $\alpha$ de Cronbach $=0,87$ e $\alpha$ de Cronbach $=0,91$ ); $\mathrm{e}$ negligência emocional $(\alpha$ de Cronbach $=0,81$ e $\alpha$ de Cronbach $=0,83$ ); por último, relativamente ao fator negligência física evidenciou uma pobre consistência interna, $(\alpha$ de Cronbach $=0,52$ e $\alpha$ de Cronbach $=0,49$ para o T1 e T2, respetivamente. Por esta razão este fator não foi considerado nas análises seguintes.

Escala de Vergonha Externa (Others As Shamers OAS; Goss et al., 1994; Tradução e adaptação: Lopes, Pinto-Gouveia, \& Castilho, 2005). É um instrumento de autorresposta que avalia a perceção que cada pessoa tem acerca da forma como os outros a vêm. Foi utilizada a versão longa constituída por 18 itens que traduzem sentimentos ou vivências de vergonha, aos quais o indivíduo deve assinalar a sua frequência, utilizando, para o efeito, uma escala de 5 pontos. Quanto maior é a pontuação, maior o nível de vergonha externa. Relativamente às características psicométricas, a versão inglesa original e a versão portuguesa para adolescentes mostraram uma boa consistência interna (Cunha et al., 2012; Goss et al., 1994). No presente estudo, este instrumento evidenciou igualmente boas qualidades psicométricas, revelando valores excelentes de consistência internam ( $\alpha$ de Cronbach $=0,95$ no T1 e $\alpha$ de Cronbach = 0,96 no T2).

\section{Procedimentos}

O presente estudo foi submetido à aprovação da Direção Geral da Educação e da Comissão Nacional de Proteção de Dados. Foram solicitados os consentimentos informados dos participantes e respetivos encarregados de educação ou representantes legais, tendo seguido os requisitos éticos e deontológicos de investigação. $\mathrm{O}$ protocolo de avaliação foi aplicado em grupo, em contexto de sala de aula, em dois momentos diferenciados, Dezembro de 2014 e 6 meses depois. De referir ainda que este estudo faz parte de uma investigação mais ampla sobre fatores preditores da depressão na adolescência (ainda em curso).

\section{Análise Estatística}

A investigação referida obedece a um desenho longitudinal, recorrendo a medições repetidas ao longo do tempo de forma avaliar as mudanças intraindividuais e utilizando uma metodologia de investigação quantitativa, não experimental, correlacional e observacional.

No que diz respeito ao tratamento dos dados, foram realizadas análises estatísticas descritivas e inferenciais utilizando para o efeito o programa SPSS (Statistic Package for the Social Sciences, versão 24). Foram utilizados diferentes testes estatísticos, selecionados em função dos objetivos das análises, das características das variáveis consideradas, bem como após a verificação dos pressupostos necessários.

Iniciou-se o processo da análise de dados através de medidas descritivas dos dados. Esta permitiu resumir a informação numérica de uma maneira estruturada, a fim de obter uma visão geral das variáveis medidas numa amostra: frequências absolutas e relativas; medidas de tendência central, como a média, e medidas de dispersão como o desvio padrão. Procedeu-se à análise da consistência interna dos instrumentos em estudo, através do cálculo do alfa de Cronbach, considerada uma estimativa adequada da fidelidade de um teste e qualidade dos itens (Marôco, 2011).

$\mathrm{Na}$ análise da estabilidade absoluta foi utilizada o coeficiente de correlação de Pearson e o teste $t$ para 
amostras emparelhadas na comparação das médias da mesma amostra em função dos tempos de avaliação.

Posteriormente, foram realizadas análises de regressão linear múltipla hierárquica para averiguar a contribuição independente dos diferentes fatores preditores e relacionais na explicação dos sintomas depressivos nos adolescentes. Foram averiguados os pressupostos para a realização destas análises, sendo cumprido o critério do tamanho da amostra e da ausência de multicolineridade (todas as variáveis independentes apresentaram valores de tolerância superiores a 0,10 e VIF inferiores a 10) (Tabachnick \& Fidell, 2007). Apesar de nem todas as variáveis seguirem uma distribuição normal (o pressuposto de normalidade foi analisado através do teste de Kolmogorov-Smirnov), os valores de assimetria e achatamento não evidenciaram graves enviesamentos. Segundo Kline (2005), valores de assimetria $|<3|$ e de achatamento $\mid<$ 10| são aceitáveis. A classificação das correlações seguiu a tipologia de Pallant (2013), diferenciando correlação baixa (valores de $r$ entre 0,10 e 0,29), correlação moderada ( $r$ entre 0,30 e 0,49$)$ e correlação elevada $(r$ entre 0,50 e 1,0 ).

\section{RESULTADOS}

Recorreu-se à comparação dos valores médios obtidos no T1 e T2, controlando o género masculino e feminino, com o objetivo de analisar a evolução das varáveis em estudo (sintomas depressivos, vivências de vergonha e experiências traumáticas).

Os valores médios relativos à estabilidade absoluta das variáveis nos dois tempos de avaliação são apresentados na Tabela 1 .

Da leitura da Tabela 1, podemos constatar que a maioria das variáveis apresenta uma estabilidade absoluta ao longo dos dois tempos de observação, sendo apenas exceção a variável referente aos sentimentos de vergonha, tendo, neste caso, os participantes indicado valores mais elevados de vergonha no segundo momento, comparativamente ao T1.

\section{Tabela 1}

Valores Médios obtidos em Função dos Tempos de Avaliação e Estabilidade Absoluta nos Instrumentos de Medida

\begin{tabular}{|c|c|c|c|c|c|c|}
\hline & \multicolumn{2}{|c|}{ Tempo 1} & \multicolumn{2}{|c|}{ Tempo 2} & \multirow{2}{*}{$t$} & \multirow{2}{*}{$p$} \\
\hline & $M$ & $D P$ & $M$ & $D P$ & & \\
\hline CDI & 11,44 & 7,33 & 10,91 & 7,12 & 1,84 & 0,067 \\
\hline Masculino & 8,85 & 5,36 & 8,13 & 5,15 & & \\
\hline Feminino & 13,10 & 7,91 & 12,67 & 7,62 & & \\
\hline OAS & 17,58 & 15,35 & 17,83 & 15,43 & $-5,17$ & $<0,001$ \\
\hline Masculino & 12,39 & 11,27 & 12,62 & 11,33 & & \\
\hline Feminino & 20,81 & 16,65 & 21,08 & 16,74 & & \\
\hline NegEmocional & 9,38 & 4,19 & & & 1,17 & 0,244 \\
\hline Masculino & 9,34 & 4,47 & 9,00 & 3,97 & & \\
\hline Feminino & 9,40 & 4,02 & 9,21 & 4,25 & & \\
\hline Abuso Afetivo & 6,72 & 2,72 & 6,48 & 2,75 & 1,75 & 0,081 \\
\hline Masculino & 6,30 & 1,90 & 6,22 & 2,44 & & \\
\hline Feminino & 6,98 & 3,10 & 6,65 & 2,92 & & \\
\hline Abuso Sexual & 5,35 & 1,70 & 5,33 & 1,73 & 0,11 & 0,910 \\
\hline Masculino & 5,23 & 0,82 & 5,22 & 1,27 & & \\
\hline Feminino & 5,42 & 2,07 & 5,41 & 1,96 & & \\
\hline Abuso Físico & 5,41 & 1,46 & 5,30 & 1,28 & 1,28 & 0,202 \\
\hline Masculino & 5,50 & 1,47 & 5,36 & 1,22 & & \\
\hline Feminino & 5,35 & 1,45 & 5,27 & 1,32 & & \\
\hline
\end{tabular}

Nota. $\mathrm{CDI}=$ Childrens's Depression Inventory; OAS = Others As Shamers; NegEmocional = Negligência emocional; $M=$ Média; $D P=$ Desvio Padrão; $t$ $=t$ Student; $p=$ nível de significância. 
Tabela 2

Correlação entre as Variáveis Vergonha e Trauma, (Tempo 1) e a Variável Sintomas Depressivos (Tempo 2)

\begin{tabular}{|c|c|c|c|c|c|}
\hline & 1 & 2 & 3 & 4 & 5 \\
\hline 1. CDI (Tempo 2) & - & & & & \\
\hline 2. OAS & $0,75^{* *}$ & - & & & \\
\hline 3. CTQ - Negligência Emocional & $0,31^{* *}$ & $0,26^{* *}$ & - & & \\
\hline 4. CTQ - Abuso Afetivo & $0,47^{* *}$ & $0,42^{* *}$ & $0,41^{* *}$ & - & \\
\hline 5. CTQ - Abuso Sexual & $0,24^{* *}$ & $0,11^{*}$ & $0,13^{*}$ & $0,32^{* *}$ & - \\
\hline 6. CTQ - Abuso Físico & $0,18^{* *}$ & $0,13^{*}$ & $0,42^{* *}$ & $0,46^{* *}$ & $0,41^{* *}$ \\
\hline
\end{tabular}

Nota. $\mathrm{CDI}=$ Childrens's Depression Inventory; OAS = Others As Shamers; CTQ = Childhood Trauma Questionnaire.

${ }^{*} p<0,05 ;{ }^{* *} p<0,01$

O estudo que se segue procurou avaliar a relação entre os acontecimentos de vida traumáticos (CTQ) e os sentimentos de vergonha (OAS) avaliados no tempo $1 \mathrm{e}$ os sintomas depressivos (CDI) medidos no tempo 2 (Tabela 2). De acordo com a Tabela 2, as variáveis vergonha e trauma (T1) e a variável sintomas depressivos (T2) estão correlacionadas entre si de forma significativa e positiva. Os sintomas depressivos apresentam uma associação elevada com a vergonha, uma correlação moderada com o abuso afetivo e fraca com a negligência emocional e abuso sexual.

Por último, para compreender qual o conjunto de variáveis que mais contribui para os predizer os sintomas depressivos nos adolescentes, foi realizada uma análise de regressão linear múltipla, utilizando as vivências de vergonha e os domínios das experiências traumáticas (experiências de abuso físico, sexual e afetivo e de negligência emocional) avaliadas no T1, como preditores (variáveis independentes) e os sintomas depressivos avaliados no T2 como variável critério (variável dependente).

Uma vez que o género mostrou uma associação significativa com os sintomas depressivos, procurámos ainda controlar a variável género, fazendo-a entrar no primeiro bloco da análise de regressão linear, depois de, previamente, transformada numa variável dummy. No segundo bloco forçámos a entrada da variável relativa às vivências de vergonha e no terceiro e último bloco, fizemos entrar as variáveis relacionadas com o trauma (Tabela 3).

\section{Tabela 3}

Análise de Regressão Linear Múltipla Utilizando o Género, as Experiências Traumáticas, Perceção de Vergonha, como Preditoras Avaliadas no Tempo 1 e os Sintomas Depressivos, Avaliados no Tempo 2

\begin{tabular}{|c|c|c|c|c|c|c|c|}
\hline Preditores & $r$ & $R^{2}$ & $F$ & $p$ & $B$ & $t$ & $p$ \\
\hline Modelo 1 & 0,31 & 0,10 & 34,25 & $<0,001$ & & & \\
\hline Género & & & & & 0,31 & 5,85 & $<0,001$ \\
\hline Modelo 2 & 0,76 & 0,58 & 218,29 & $<0,001$ & & & \\
\hline Género & & & & & 0,12 & 3,22 & $<0,001$ \\
\hline OAS & & & & & 0,72 & 19,06 & $<0,001$ \\
\hline Modelo 3 & 0,79 & 0,63 & 88,32 & $<0,001$ & & & \\
\hline Género & & & & & 0,12 & 3,33 & $<0,001$ \\
\hline OAS & & & & & 0,63 & 16,06 & $<0,001$ \\
\hline Abuso Afetivo & & & & & 0,15 & 3,38 & $<0,001$ \\
\hline Negligência Emocional & & & & & 0,10 & 2,51 & 0,013 \\
\hline Abuso Sexual & & & & & 0,12 & 3,07 & 0,002 \\
\hline Abuso Físico & & & & & $-0,06$ & $-1,26$ & 0,21 \\
\hline
\end{tabular}

Nota. OAS = Others As Shamers; $R^{2}=$ coeficiente de determinação; $B=$ valor de beta; $t=$ teste $t$ Student. 
O primeiro bloco da função de regressão contribuiu com $10 \%$ para o total da variância $\left(R^{2}=0,10\right)$, o segundo bloco contribuiu com $48,2 \%\left(R^{2}=0,58,\right)$ e o terceiro bloco acrescentou 5\% $\left(R^{2}=0,63\right)$.

A função no seu conjunto, constituída pelas vivências de vergonha, os acontecimentos traumáticos, nomeadamente abuso afetivo, abuso sexual e negligência emocional, explicou $63 \%$ do total dos sintomas depressivos, sendo um modelo preditor significativo.

Quando introduzidos os três blocos, o valor de Beta mais elevado foi para a vivência de vergonha $(\beta=0,63$; $p<0,001)$, seguindo-se o abuso afetivo $(\beta=0,15 ; p=$ $0,001)$, o género $(\beta=0,12 ; p=0,001)$, depois o abuso sexual ( $\beta=0,12 ; p=0,002)$ e, por último, a variável relativa à negligência emocional $(\beta=0,10 ; p=0,013)$. Estes resultados indicaram que o fato de pertencer ao sexo feminino, experienciar mais vivências de vergonha, e mais experiências traumáticas de abuso afetivo, abuso sexual e de negligência emocional permitem predizer mais sintomas depressivos na adolescência. Mesmo depois de controlado o efeito do género e da vergonha, as experiências traumáticas revelaram um efeito significativo e independente nos sintomas depressivos.

\section{DISCUSSÃO}

Estudos longitudinais breves, que investigam a ocorrência de determinados acontecimentos de vida, neste caso os acontecimentos traumáticos e vivências de vergonha nos adolescentes, constituem uma boa oportunidade para clarificar a psicopatologia depressiva precoce a qual pode influenciar a longo prazo o funcionamento adaptativo do indivíduo. O presente estudo pretendeu, assim, explorar de que forma diferentes tipos de acontecimentos traumáticos ocorridos ao longo da infância e sentimentos de vergonha estão associados a sintomas depressivos na adolescência avaliados em dois tempos separados por seis meses.

$\mathrm{Na}$ globalidade, os resultados corroboraram a hipótese do poder preditivo do trauma e da vergonha na evolução dos sintomas depressivos a seis meses. Todas as variáveis em estudo mostraram uma estabilidade absoluta, excluindo a vergonha externa, a qual evidenciou valores médios mais elevados no T2. Mais estudos longitudinais são necessários para poder esclarecer este resultado, já que até há data os estudos efetuados com adolescentes são ainda escassos. De notar que a investigação sobre a relação entre a vergonha e a idade tem sugerido um efeito significativo na transição para a idade adulta, e não uma variação ao longo da adolescência (Cunha, Xavier, Cherpe, \& Pinto-Gouveia, 2017; De Rubeis \& Hollenstein, 2009; Tangney \& Dearing, 2002).

Por sua vez, as variáveis associadas ao trauma e vergonha no Tempo 1 mostraram-se associadas aos sintomas depressivos avaliados no Tempo 2 (6 meses depois). O mesmo é dizer que os nossos dados indicam que quanto maior é o nível de acontecimentos traumáticos (sob a forma de experiências de abuso afetivo, físico, sexual e de negligência emocional), maior é o receio de existir na mente dos outros de forma negativa e maior é o nível de sintomas depressivos aos 6 meses. Estudos sobre a adolescência valorizam esta fase como sendo uma fase crítica, onde ocorrem sucessivas reorganizações qualitativas dentro e entre sistemas biológico, emocional, cognitivo, comportamental e social que dirigem e redirecionam o curso do desenvolvimento humano (Papalia, Olds, \& Feldman, 2006). Estudos transversais têm confirmado que a vergonha está associada positivamente com a depressão nos jovens (Andrews, Qian, \& Valentine, 2002; Åslund et al., 2007; Balsamo et al., 2015; Cunha et al., 2012; Muris \& Meesters, 2014). Por sua vez, crianças que sofreram vários episódios de abuso/maltrato são mais suscetíveis a um maior risco de psicopatologia, formando assim visões e respostas negativas que contribuem para o desenvolvimento de esquemas depressivos (Mandelli et al., 2015). Com efeito, os nossos resultados mostraram uma associação moderada ao longo de seis meses entre o abuso afetivo e negligência emocional e os sintomas depressivos. As dinâmicas da situação abusiva na infância, especificamente na forma de abuso afetivo/emocional ou negligência emocional têm sido progressivamente estudadas em pesquisas transversais e prospetivas, indicando uma forte relação com desenvolvimento de depressão clínica na adolescência, e subsequentemente, na vida adulta (Dunn, McLaughlin, Slopen, Rosand, \& Smoller, 2013; Infurna, Rivers, Reich, \& Zautra, 2015; Price, Higa-McMillan, \& Frueh, 2013). No entanto, a maior parte da investigação existente enfatiza o abuso sexual (Bonanno et al., 2002; Mandelli et al., 2015; Musliner \& Singer, 2014) e o abuso físico como fator de risco para a depressão em adultos (Infurna et al., 2015; Liu et al., 2009). Contudo, na nossa investigação os resultados evidenciaram uma associação baixa, salientando-se uma associação maior com as experiências de abuso afetivo e de negligência emocional. A interpretação destes resultados exige 
cautela e a necessidade de ter em conta o facto da presente amostra ser da comunidade, e não uma amostra clínica ou mista, o que pode ajudar a explicar algumas diferenças encontradas relativamente ao peso do tipo de experiências traumáticas.

Por último, na análise do conjunto de variáveis que melhor explica os sintomas depressivos nos adolescentes, os nossos resultados mostraram um modelo preditor significativo constituído pelas vivências de vergonha externa, pelos acontecimentos traumáticos, nomeadamente abuso afetivo, abuso sexual e negligência emocional e pela variável género. Ou seja, receios mais elevados de que os outros os vejam como incompetentes, defeituosos ou inferiores e mais experiências adversas ocorridas na infância, nomeadamente de abuso afetivo, abuso sexual e negligência emocional, bem como pertencer ao género feminino predizem mais sintomas depressivos a seis meses. A vergonha externa, isto é, a forma como os adolescentes percecionam que os outros os vêm, mostrou-se o preditor mais forte, contribuindo com $48 \%$ da variância explicada. Contudo, mesmo depois de controlado o efeito do género e da vergonha, as experiências traumáticas têm ainda uma relação significante e independente com os sintomas depressivos.

Estes resultados são confirmados pela literatura internacional que analisou a relação entre os efeitos do abuso durante a infância e os efeitos a médio e longo prazo, sendo esta moderada por diferentes variáveis como o tipo de abuso, intensidade, duração e género (Infurna et al, 2015; Sousa et al., 2011).

De acordo com a investigação, os sentimentos de vergonha estão relacionados com histórias de abuso e com várias formas de maus tratos psicológicos (indiferença, rejeição, abandono, negligência por omissão às necessidades básicas da criança) (Slep, Heyman, \& Snarr, 2011; Szentágotai-Tătar et al., 2015), bem como se têm mostrado preditores de sintomas depressivos (Gibb et al., 2001). O género é também considerado, na maioria das investigações, uma variável preditora de depressão, uma vez que, existem diferenças na prevalência e manifestação de sintomas depressivos entre os rapazes e raparigas, no início da adolescência (English, Widom, \& Brandford, 2004; Rosso et al., 2014). Por sua vez, estudos longitudinais demonstram que a exposição a um único tipo de mau trato ou a vários tipos está relacionada com o aumento da internalização e externalização de comportamentos na infância e adolescência (Moylan et al., 2010).
O facto de os nossos dados terem sido obtidos com recurso a um desenho longitudinal e prospetivo vem reforçar a ideia que variáveis preditoras (eventos traumáticos e vivências de vergonha) são importantes antecedentes dos sintomas depressivos. Na verdade, o presente estudo pode acrescentar informações que esclarecem a importância relativa de mecanismos subjacentes à vulnerabilidade aos sintomas depressivos. Não obstante este ponto forte, sofre de limitações diversas. A principal prende-se com a utilização exclusiva da população geral, sendo importante estender a outra amostras clínicas de adolescentes. Seria igualmente interessante recorrer a outras formas de avaliação (por exemplo, entrevistas clínicas, medidas fisiológicas) e outros informadores (e.g., familiares), ultrapassando, assim, a dependência absoluta de questionários de autorrelato, dada o conhecido efeito da elevada desejabilidade social nos adolescentes. Consideramos ainda crucial aumentar momentos ao longo do tempo para recolha de dados e ver a estabilidade inter e intraindividual.

Em síntese, a presente investigação contribuiu para a importância de uma sinalização precoce nas crianças/adolescentes, principalmente do sexo feminino, para iniciar uma intervenção preventiva e interventiva dirigida a este tipo de experiências adversas que permitam minimizar o seu impacto.

Conflito de interesses | Conflict of interest: Nenhum | None. Fontes de financiamento | Funding sources: Nenhuma | None.

\section{REFERÊNCIAS}

Ahmed, S. P., Bittencourt-Hewitt, A., \& Sebastian, C. L. (2015). Neurocognitive bases of emotion regulation development in adolescence. Developmental Cognitive Neuroscience, 15, 11-25. https://doi.org/10.1016/j.dcn.2015.07.006

Andrews, B., Qian, M., \&Valentine, D. J. (2002). Predicting depressive symptoms with a new measure of shame: The Experience of Shame Scale. British Journal of Clinical Psychology, 41(1), 29-42. https://doi.org/10.1348/014466502163778

Åslund, C., Nilsson, K. W., Starrin, B., \& Sjöberg, R. L. (2007). Shaming experiences and the association between adolescent depression and psychosocial risk factors. European Child and Adolescent Psychiatry, 16(5), 298-304. https://doi.org/10.1007/s00787-0060564-1

Balsamo, M., Macchia, A., Carlucci, L., Picconi, L., Tommasi, M., Gilbert, P., \& Saggino, A. (2015). Measurement of external shame: An inside view. Journal of Personality Assessment, 97(1), 81-89. https://doi.org/10.1080/00223891.2014.947650

Barry, M., Murphy, M., \& O'Donovan, H. (2017). Assessing the effectiveness of a cognitive behavioural group coaching intervention in reducing symptoms of depression among adolescent males in a school setting. International Coaching 
Psychology Review, 12(2), 101-109. Retrieved from http://psycnet.apa.org/record/2018-01291-003

Bennett, D. S., Sullivan, M. W., \& Lewis, M. (2010). Neglect children, shame-proneness, and depressive symptoms. Child Maltreatment, 15(4), 305-314. https://doi.org/10.1177/1077559510379634

Bernstein, D. P., \& Fink, L. (1998). Childhood Trauma Questionnaire: A retrospective self-report manual. San Antonio, TX: The Psychological Corporation.

Bonanno, G. A., Keltner, D., Noll, J. G., Putnam, F. W., Trickett, P. K., Lejeune, J., \& Anderson, C. (2002). When the face reveals what words do not: Facial expressions of emotion, smiling, and the willingness to disclose childhood sexual abuse. Journal of Personality and Social Psychology, 83(1), 94-110. https://doi.org/10.1037/0022-3514.83.1.94

Bernstein, D. P., Stein, J. A., Newcomb, M. D., Walker, E., Pogge, D., Ahluvalia, T., ... Zule, W. (2003). Development and validation of a brief screening version of the Childhood Trauma Questionnaire. Child Abuse \& Neglect, 27(2), 169-190. https://doi.org/10.1016/S0145-2134(02)00541-0

Cardoso, P. M. S., Rodrigues, C., \& Vilar, A. (2004). Prevalência de sintomas depressivos em adolescentes portugueses [Prevalence of depressive symptoms in Portuguese adolescents]. Análise Psicológica, 22(4), 667-675. https://doi.org/10.14417/ap.264

Cherpe, S., Matos, A. P., \& Pereira, A. (2010). Versão Portuguesa do Childhood Trauma Questionnaire [Portuguese version of the Childhood Trauma Questionnaire]. Manuscrito não publicado, Faculdade de Psicologia e Ciências da Educação da Universidade de Coimbra, Portugal.

Costello, E. J., Copeland, W., \& Angold, A. (2011). Trends in psychopathology across the adolescent years: What changes when children become adolescents, and when adolescents become adults? Journal of Child Psychology and Psychiatry, 52(10), 1015-1025. https://doi.org/10.1111/j.1469-7610.2011.02446.x

Cunha, M., Matos, M., Faria, D., \& Zagalo, S. (2012). Shame memories and psychopathology in adolescence: The mediator effect of shame. International Journal of Psychology \& Psychological Therapy, 12(2), 203-218. Retrieved from http://www.ijpsy.com/volumen12/num2/327/shame-memoriesand-psychopathologyin-adolescence-EN.pdf

Cunha, M., Xavier, A. M. J., Cherpe, S., \& Pinto-Gouveia, J. (2017) Assessment of shame in adolescents: The 'Other as Shamer' Scale. Psicologia: Teoria e Pesquisa, 33, 1-9. https://doi.org/10.1590/0102.3772e3336

Dahl, R. E. (2004). Adolescent brain development: A period of vulnerabilities and opportunities. Keynote address. Annals of the New York Academy of Sciences, 1021(1), 1-22. https://doi.org/10.1196/annals.1308.001

De Rubeis, S., \& Hollenstein, T. (2009). Individual differences in shame and depressive symptoms during early adolescence. Personality and Individual Differences, 46(4), 477-482. https://doi.org/10.1016/j.paid.2008.11.019

Dunn, E. C., McLaughlin, K. A., Slopen, N., Rosand, J., \& Smoller, J. W (2013). Developmental timing of child maltreatment and symptoms of depression and suicidal ideation in young adulthood: Results from the National Longitudinal Study on Adolescent Health. Depression and Anxiety, 30(10), 955-964. https://doi.org/10.1002/da.22102

English, D. J., Widom, C. S., \& Brandford, C. (2004). Another look at the effects of child abuse. National Institute of Justice Journal, 251, 2324. Retrieved from https://www.ncjrs.gov/pdffiles1/jr000251g.pdf

Feiring, C. (2005). Emotional development, shame, and adaptation to child maltreatment. Child Maltreatment, 10(4), 307-310. https://doi.org/10.1177/1077559505281307
Firth, M. T. (2014). Childhood abuse and depressive vulnerability in clients with gender dysphoria. Counselling and Psychotherapy Research, 14(4), 297-305. https://doi.org/10.1080/14733145.2013.845236

Grassi-Oliveira, R., Stein, L. M., \& Pezzi, J. C. (2006). Tradução e validação de conteúdo da versão em português do Childhood Trauma Questionnaire [Translation and content validation of the Childhood Trauma Questionnaire into Portuguese language]. Revista de Saúde Pública, 40(2) 249-255. https://doi.org/10.1590/S0034-89102006000200010

Gibb, B. E., Alloy, L. B., Abramson, L. Y., Rose, D. T., Whitehouse, W. G., Donovan, P., ... Tierney, S. (2001). History of childhood maltreatment, negative cognitive style, and episodes of depression in adulthood. Cognitive Therapy and Research, 25(4), 425-446. Retrieved from https://sites.temple.edu/moodandcognitionlab/files/2014/01/G ibb-et-al.-2001a.pdf

Gilbert, P. (1998). What is shame? Some core issues and controversies. In P. Gilbert \& B. Andrews (Eds.), Shame: Interpersonal behavior, psychopathology, and culture (pp. 3-38). New York, NY: Oxford University Press.

Gilbert, P. (2002). Understanding the biopsychosocial approach: Conceptualization. Clinical Psychology, 14, 13-17. Retrieved from http://www.critpsynet.freeuk.com/Gilbert.htm

Gilbert, P. (2006). A biopsychosocial and evolutionary approach to formulation with a special focus on shame. In N. Tarrier (Ed.), Case formulation in cognitive behaviour therapy: The treatment of challenging and complex cases (pp. 81-112). New York, NY: Routledge/Taylor \& Francis Group.

Gilbert, P., \& Irons, C., (2009). Shame, self-criticism, and selfcompassion in adolescence. In N. Allen (Ed.), Psychopathology in adolescence. Cambridge: Cambridge University Press.

Gore, F. M., Bloem, P. J., Patton, G. C., Ferguson, J., Joseph, V., Coffey, C., ... Mathers, C. D. (2011). Global burden of disease in young people aged 10-24 years: A systematic analysis. The Lancet, 377(9783), 2093-2102. https://doi.org/10.1016/S01406736(11)60512-6

Goss, K., Gilbert, P., \& Allan, S. (1994). An exploration of shame measures - I: The Other as Shamer Scale. Personality and Individual Differences, 17(5), 713-717. https://doi.org/10.1016/01918869(94)90149-X

Hopwood, C. J., Ansell, E. B., Fehon, D. C., \& Grilo, C. M. (2011). The mediational significance of negative/depressive affect in the relationship of childhood maltreatment and eating disorder features in adolescent psychiatric inpatients. Eating and Weight Disorders - Studies on Anorexia, Bulimia and Obesity, 16(1), e9e16. https://doi.org/10.1007/BF03327515

Infurna, F. J., Rivers, C. T., Reich, J., \& Zautra, A. J. (2015). Childhood trauma and personal mastery: Their influence on emotional reactivity to everyday events in a community sample of middleaged adults. PLOS ONE, 10(4), e0121840. https://doi.org/10.1371/journal.pone.0121840

Irons, C., \& Gilbert, P. (2005). Evolved mechanisms in adolescent anxiety and depression symptoms: The role of the attachment and social rank systems. Journal of Adolescence, 28(3), 325-341. https://doi.org/10.1016/j.adolescence.2004.07.004

Kim, J., \& Cicchetti, D. (2006). Longitudinal trajectories of self-system processes and depressive symptoms among maltreated and nonmaltreated children. Child Development, 77(3), 624-639. https://doi.org/10.1111/j.1467-8624.2006.00894.x

Kim, J., Talbot, N. L., \& Cicchetti, D. (2009). Childhood abuse and current interpersonal conflict: The role of shame. Child Abuse \& Neglect, 33(6), 362-371. https://doi.org/10.1016/j.chiabu.2008.10.003

Kline, R. B. (2005). Principles and practice of structural equation modeling (2nd ed.). New York, NY: Guilford Press. 
Kovacs, M. (1985). The Children's Depression Inventory (CDI). Psychopharmacology Bulletin, 21(4), 995-998.

Liu, R. T., Alloy, L. B., Abramson, L. Y., lacoviello, B. M., \& Whitehouse, W. G. (2009). Emotional maltreatment and depression: Prospetive prediction of depressive episodes. Depression and Anxiety, 26(2), 174-181. https://doi.org/10.1002/da.20545

Lopes, B., Pinto-Gouveia, J., \& Castilho, P. (2005). Portuguese version of the Others as Shamer Scale. Unpublish manuscript.

Mandelli, L., Petrelli, C., \& Serretti, A. (2015). The role of specific early trauma in adult depression: A meta-analysis of published literature. Childhood trauma and adult depression. European Psychiatry, 30(6), 665-680. https://doi.org/10.1016/j.eurpsy.2015.04.007

Marôco, J. (2011). Análise estatística com o SPSS Statistics [Statistical analysis with SPSS Statistics] (5th ed.). Pero Pinheiro: Report Number.

Matos, M., \& Pinto-Gouveia, J. (2010). Shame as a traumatic memory. Clinical Psychology and Psychotherapy, 17(4), 299-312. https://doi.org/10.1002/cpp.659

Matos, M., Pinto-Gouveia, J., \& Duarte, C. (2012). When I don't like myself: Portuguese version of the internalized shame scale. The Spanish Journal of Psychology, 15(3), 1411-1423. https://doi.org/10.5209/rev_SJOP.2012.v15.n3.39425

Matos, M., Pinto-Gouveia, J., \& Duarte, C. (2013). Internalizing early memories of shame and lack of safeness and warmth: The mediating role of shame on depression. Behavioural and Cognitive Psychotherapy, 41(4), 479-493. https://doi.org/10.1017/S1352465812001099

Marujo, H. (1994). Síndromas depressivos na infância e na adolescência [Depressive syndromes in childhood and adolescence] (Unpublished doctoral dissertation). Faculdade de Psicologia e Ciências da Educação da Universidade de Lisboa, Lisboa, Portugal.

McLean, K. C., Breen, A. V., \& Fournier, M. A. (2010). Constructing the self in early, middle, and late adolescent boys: Narrative identity, individuation, and well-being. Journal of Research on Adolescence, 20(1), 166-187. https://doi.org/10.1111/j.15327795.2009.00633.x

Monteiro, S., Matos, A. P., \& Oliveira, S. (2015). The moderating effect of gender: Traumatic experiences and depression in adolescence. Procedia - Social and Behavioral Sciences, 165, 251-259. https://doi.org/10.1016/j.sbspro.2014.12.629

Moylan, C. A., Herrenkohl, T. I., Sousa, C., Tajima, E. A., Herrenkohl, R. C., \& Russo, M. J. (2010). The effects of child abuse and exposure to domestic violence on adolescent internalizing and externalizing behavior problems. Journal of Family Violence, 25(1), 53-63. https://doi.org/10.1007/s10896-009-9269-9

Muris, P., Meesters, C., Heijmans, J., van Hulten, S., Kaanen, L., Oerlemans, B., ... Tielemans, T. (2016). Lack of guilt, guilt, and shame: A multi-informant study on the relations between selfconscious emotions and psychopathology in clinically referred children and adolescents. European Child \& Adolescent Psychiatry, 25(4), 383-396. https://doi.org/10.1007/s00787-0150749-6

Muris, P., \& Meesters, C. (2014). Small or big in the eyes of the other: On the developmental psychopathology of self-conscious emotions as shame, guilt, and pride. Clinical Child and Family $\begin{array}{lll}\text { Psychology Review, 17(1), 19-40 } & \end{array}$ https://doi.org/10.1007/s10567-013-0137-z

Musliner, K. L., \& Singer, J. B. (2014) Emotional support and adult depression in survivors of childhood sexual abuse. Child Abuse \& Neglect, 38(8), 1331-1340. https://doi.org/10.1016/j.chiabu.2014.01.016
Negrao, C., Bonanno, A. G., Noll, J. G., Putnam, F. W., \& Trickett, P. K. (2005). Shame, humiliation, and childhood sexual abuse: Distinct contributions and emotional coherence. Child Maltreatment, 10(4), 350-363. https://doi.org/10.1177/1077559505279366

Papalia, D. E., Olds, S. W., \& Feldman, R. D. (2006). Desenvolvimento Humano (8th ed.). Porto Alegre: Artmed.

Pallant, J. (2013). SPSS survival manual: A step by step guide to data analysis using IBM SPSS (5th ed.). Crows Nest, NSW: Allen \& Unwin.

Pereira, I. M., Matos, A. P., \& Azevedo, A. (2014). Versão portuguesa do Questionário de Pensamentos Automáticos-Revisto: Relação com sintomatologia depressiva em adolescentes [Portuguese version of the automatic thoughts questionnaires - revised: Relation with depressive symptomatology in adolescents]. Psicologia, Saúde \& Doenças, 15(1), 36-46. https://doi.org/10.15309/14psd150105

Pinquart, M., \& Shen, Y. (2011). Depressive symptoms in children and adolescents with chronic physical illness: An updated metaanalysis. Journal of Pediatric Psychology, 36(4), 375-384. https://doi.org/10.1093/jpepsy/jsq104

Price, M., Higa-McMillan, C., Kim, S., \& Frueh, C. (2013). Trauma experience in children and adolescents: An assessment of the effects of trauma type and role of interpersonal proximity. Journal of Anxiety Disorders, 27(7), 652-660. https://doi.org/10.1016/j.janxdis.2013.07.009

Rosso, A. D., Beber, S., Bianco, F., Gregorio, D. D., Paolo, M. D., Lauriola, A. L., ... Basile, B. (2014). La vergogna in psicopatologia [Shame in psychopathology]. Cognitivismo Clinico, 11(1), 27-61. Retrieved from http://www.fioriti.it/riviste/pdf/2/14-1DelRossoweb.pdf

Shore, L., Toumbourou, J. W., Lewis, A. J., \& Kremer, P. (2018). Longitudinal trajectories of child and adolescent depressive symptoms and their predictors - A systematic review and metaanalysis. Child and Adolescent Mental Health, 23(2), 107-120. https://doi.org/10.1111/camh.12220

Slep, A. M. S., Heyman, R. E., \& Snarr, J. D. (2011). Child emotional agression and abuse: Definitions and prevalence. Child Abuse \& Neglect, 35(10), 783-796. https://doi.org/10.1016/j.chiabu.2011.07.002

Sousa, C., Herrenkohl, T. I., Moylan, C. A., Tajima, E. A., Klika, J. B., Herrenkohl, R. C., \& Russo, M. J. (2011). Longitudinal study on the effects of child abuse and children's exposure to domestic violence, parent-child attachments, and antisocial behavior in adolescence. Journal Interpersonal Violence, 26(1), 111-136. https://doi.org/10.1177/0886260510362883

Spinhoven, P., Penninx, B. W., Hickendorff, M., van Hemert, A. M., Bernstein, D. P., \& Elzinga, B. M. (2014). Childhood Trauma Questionnaire: Factor structure, measurement invariance, and validity across emotional disorders. Psychological Assessment, 26(3), 717-729. https://doi.org/10.1037/pas0000002

Steinberg, L. (2005). Cognitive and affective development in adolescence. Trends in Cognitive Sciences, 9(2), 69-74. https://doi.org 10.1016/j.tics.2004.12.005

Stuewing, J., \& McCloskey, L. A. (2005). The relation of child maltreatment to shame and guilt among adolescents: Psychological routes to depression and delinquency. Child Maltreatment, 10(4), 324-336. https://doi.org/10.1177/1077559505279308

Steinberg, L. (2010). A behavioral scientist looks at the science of adolescent brain development. Brain and Cognition, 72(1), 160164. https://doi.org/10.1016/j.bandc.2009.11.003

Tabachnick, B. G., \& Fidell, L. S. (2007). Using multivariate statistics (5th ed.). Boston: Pearson Education Inc.

Tangney, J. P., \& Dearing, R. L. (2002). Shame and guilt (1st ed.). New York, NY: Guilford Press. 
Thapar, A., Collishaw, S., Pine, D. S., \& Thapar, A. K. (2012). Depression in adolescence. The Lancet, 379(9820), 1056-1067. https://doi.org/10.1016/S0140-6736(11)60871-4

Szentágotai-Tătar, A., Chis, A., Vulturar, R., Dobrean, A., Cândea, D. M., \& Miu, A. C. (2015). Shame and guilt-proneness in adolescents: Gene-environment interactions. PLOS ONE, 10(7), e0134716. https://doi.org/10.1371/journal.pone.0134716
Tolchinsky, A. (2014). Acute trauma in adulthood in the context of childhood traumatic experiences. Neuropsychoanalysis: An Interdisciplinary Journal for Psychoanalysis and the Neurosciences, 16(2), 129-137. https://doi.org/10.1080/15294145.2014.963645

World Health Organization. (2018). Adolescents: Health risks and solutions. Retrieved from 